\title{
Adaptation, mise à l'épreuve et évaluation d'interventions complexes en santé publique : leçons tirées du Nurse-Family Partnership dans le secteur de la santé publique au Canada
}

\author{
S. M. Jack, I.A., Ph. D. (1, 2, 3); N. Catherine, Ph. D. (4); A. Gonzalez, Ph. D. (2, 5); H. L. MacMillan, M.D. (2, 3, 5, 6); \\ D. Sheehan, I.A., M. Serv. Soc. (4); C. Waddell, M.D. (4); pour le groupe scientifique du British Columbia Healthy \\ Connections Project
}

Cet article a fait l'objet d'une évaluation par les pairs.

Diffuser cet article sur Twitter

\section{Résumé}

Introduction : Le Nurse-Family Partnership (NFP) est un programme de visites à domicile destiné aux nouvelles jeunes mères défavorisées sur le plan socioéconomique. Les données issues de trois essais contrôlés randomisés (ECR) américains ont solidement démontré l'efficacité des interventions quant à l'amélioration de l'issue de la grossesse, de la santé et du développement des enfants ainsi que de l'autonomie économique des mères. Cependant, l'efficacité du NFP dans le contexte canadien des services de santé et des services sociaux, qui diffère de celui des États-Unis, reste à déterminer. Cet article vise à décrire le processus complexe suivi pour adapter la recherche sur le NFP et mettre ainsi en œuvre ce programme au Canada.

Méthodologie : L'évaluation menée au Canada se divise en trois étapes : 1) adaptation de l'intervention, 2) mise à l'épreuve de l'intervention dans des études de faisabilité et d'acceptabilité à petite échelle et 3) réalisation d'un ECR et d'une évaluation du processus dans le cadre de l'étude intitulée British Columbia Healthy Connections Project $(\mathrm{BCHCP})$. Cette évaluation à grande échelle permettra d'enrichir la base de données probantes du NFP par la tenue d'une étude supplémentaire sur les mécanismes biologiques susceptibles de témoigner de la relation entre l'intervention et les effets sur le comportement des enfants.

Résultats : L'adaptation de la documentation du NFP pour les visites à domicile est un processus continu. Un projet pilote a montré la faisabilité du recrutement des femmes admissibles au NFP. Il a aussi révélé qu'il était préférable au Canada que le NFP soit mis en œuvre par les organismes de santé publique et que les infirmières et infirmiers en santé publique (ISP) s'occupent des interventions. Enfin, il a montré que ce programme intensif de visites à domicile a bénéficié d'une réception positive de la part des clientes, des membres de leur famille et des fournisseurs de soins de santé.

Les prochaines étapes - à savoir l'ECR et l'évaluation du processus - ont été entamées dans le cadre du BCHCP. Ce projet jettera les bases d'une évaluation à long terme des principaux résultats en matière de santé publique concernant des familles hautement vulnérables.

Mots-clés : études d'intervention, prévention, soins infirmiers de santé publique, visites à domicile, violence envers les enfants
Principales constatations

- L'efficacité du programme Nurse-Family Partnership, une intervention de santé publique complexe qui améliore la vie des nouvelles mères vulnérables et de leur famille, a été prouvée aux États-Unis.

- Avant d'être mise en œuvre au Canada, elle doit être adaptée, mise à l'épreuve et évaluée en fonction du contexte, tant du point de vue des résultats que du processus.

\section{Introduction}

Offrir des interventions de santé publique fondées sur des données probantes et destinées à favoriser la santé et à prévenir les problèmes de santé et les problèmes sociaux est un projet qui nécessite un processus complexe englobant l'élaboration, la mise à l'épreuve et l'évaluation des interventions et des programmes prometteurs. Cet article vise à décrire le processus à plusieurs étapes amorcé au Canada pour adapter, mettre à l'épreuve et évaluer le programme de visites à domicile NurseFamily Partnership (NFP). Ce programme en santé publique s'est révélé efficace aux États-Unis pour améliorer l'issue des grossesses, la santé et le développement des enfants ainsi que l'autonomie économique des nouvelles mères défavorisées sur le

Rattachement des auteurs :

1. École de soins infirmiers, Université McMaster, Hamilton (Ontario), Canada

2. Centre d'études de l'enfance Offord, Université McMaster, Hamilton (Ontario), Canada

3. Département d'épidémiologie clinique et de biostatistique, Université McMaster, Hamilton (Ontario), Canada

4. Centre des politiques sur la santé des enfants, Université Simon Fraser, Vancouver (Colombie-Britannique), Canada

5. Département de psychiatrie et des neurosciences comportementales, Université McMaster, Hamilton (Ontario), Canada

6. Département de pédiatrie, Université McMaster, Hamilton (Ontario), Canada

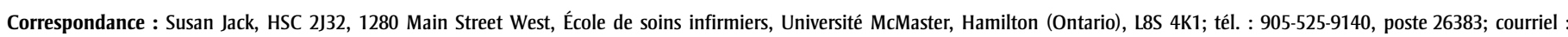
jacksm@mcmaster.ca 
plan socioéconomique et de leurs enfants. Cependant, l'efficacité de ce programme n’a pas encore été vérifiée dans le contexte de la santé publique et des services sociaux du Canada. La mesure et l'amélioration des principaux résultats en santé mentale, en particulier la prévention de la violence et de la négligence envers les enfants, est l'un des aspects les plus importants de l'évaluation et de la mise en œuvre du NFP au Canada.

Malgré les efforts déployés pour la freiner, la violence envers les enfants, qui englobe toutes les formes de maltraitance et de négligence, reste un problème de santé publique majeur au Canada. Selon une enquête nationale récente, environ le tiers des Canadiens et Canadiennes ont vécu une ou plusieurs formes de violence durant leur enfance ${ }^{1}$. Afifi et ses collaborateurs ${ }^{1}$ ont aussi signalé l'existence d'une relation dose-réponse entre, d'une part, l'exposition à la violence en tant qu'enfant qui la subit et, d'autre part, l'apparition de troubles de santé mentale.

Les visites à domicile sont encouragées depuis plus de 30 ans pour prévenir la maltraitance et la négligence envers les enfants. Durant les années 1980, à la lumière des résultats de l'essai pilote du programme Hawaii Healthy Start ${ }^{2}$, les visites à domicile effectuées par des paraprofessionnels se sont largement répandues au Canada et aux ÉtatsUnis : elles étaient considérées comme le principal moyen de prévenir la violence envers les enfants. Toutefois, au cours des deux décennies suivantes, le recours croissant à des méthodes rigoureuses d'évaluation des résultats a clairement montré que les programmes de visite à domicile n'étaient pas tous également efficaces en ce qui concerne la prévention de la violence envers les enfants et des problèmes connexes comme les blessures. Le NFP est celui qui a donné les meilleurs résultats en matière de prévention de la maltraitance et de la négligence envers les enfants, s'il on se fie aux cas déclarés par les services de protection de la jeunesse et aux problèmes connexes comme les blessures ${ }^{3}$.

Le NFP est un programme destiné aux nouvelles mères défavorisées sur le plan socioéconomique. Il commence durant la grossesse et se poursuit jusqu'aux 2 ans de l'enfant. Les trois essais contrôlés randomisés (ECR) américains ont montré que le NFP comporte de nombreux avantages pour la santé maternelle et infantile, notamment la prévention de la maltraitance et de la négligence envers les enfants ${ }^{4}$. Plus récemment, les résultats du suivi des ECR effectués à Memphis ont fait ressortir une réduction de la mortalité toutes causes confondues chez les mères ayant bénéficié de ces visites ainsi qu'une réduction de la mortalité infantile évitable chez les enfants une fois atteint l'âge de 20 ans $^{5}$. En 2004, en se basant sur les données issues des trois ECR, le Washington State Institute for Public Policy a estimé le retour sur l'investissement pour la société à 17000 \$US par famille participant au NFP 6

\section{Méthodologie}

À la lumière de ces résultats encourageants, des efforts visant à mettre en œuvre le NFP au Canada ont commencé il y a plus de 10 ans dans le but de prévenir la violence envers les enfants, d'améliorer leur santé mentale et leur développement et de faciliter la vie des nouvelles mères défavorisées. Cependant, les constatations positives faites aux États-Unis ne sont pas nécessairement reproductibles au Canada, compte tenu des différences politiques, socioéconomiques, démographiques et géographiques qui existent entre les deux pays. Par exemple, le Canada offre des programmes publics plus généreux, notamment en ce qui concerne les soins de santé, le soutien au revenu et les prestations pour enfants, et les mères à faible revenu y bénéficient d'une couverture de soins quasi universelle. C'est pourquoi le $\mathrm{D}^{\mathrm{r}}$ David Olds, concepteur du programme, exige que le NFP, avant d'être mis en œuvre à l'extérieur des
États-Unis, fasse l'objet de travaux visant à déterminer son efficacité dans le nouveau pays.

Olds et ses collaborateurs ${ }^{7}$ sont déterminés à adapter le NFP pour des pays autres que les États-Unis pourvu que les résultats fassent l'objet d'une évaluation rigoureuse confirmant que le programme est bénéfique pour les mères défavorisées et leurs enfants. Le Centre de recherche sur la prévention de l'Université du Colorado à Denver exige des pays étrangers (autres que les États-Unis) mettant en œuvre le NFP qu'ils suivent le protocole en quatre étapes ${ }^{8}$ présenté dans le tableau 1. Ce processus a par exemple été respecté lorsque le programme Family Nurse Partnership a été lancé et évalué en Angleterre: d'abord, une évaluation formative, qui visait à assurer la fidélité aux éléments de modèle, a permis de documenter la mise à l'épreuve de l'intervention dans 10 sites pilotes $^{9}$. Cette étude a été suivie d'un ECR à grande échelle ayant pour but de déterminer l'efficacité globale du programme et dont les résultats sont attendus en 2015. Aux PaysBas, où le NFP a été rebaptisé VoorZorg, des mesures semblables ont été prises pour traduire et adapter les lignes directrices américaines en fonction du contexte local. Elles ont été suivies d'une étude pilote sur l'intégrité du traitement et sur la faisabilité du programme dans le pays ${ }^{10}$. Une fois ces travaux terminés, on a réalisé un ECR dont les résultats ont confirmé l'efficacité supérieure du NFP dans la réduction de la violence conjugale chez les mères visitées à domicile, comparativement aux soins habituels ${ }^{11}$.

Le tableau 2 présente la chronologie de toutes les activités liées à l'adaptation, à la mise à l'épreuve et à l'évaluation du NFP au Canada.

TABLEAU 1

Protocole de reproduction et d'évaluation du Nurse-Family Partnership à l'échelle internationale

1. Adapter le NFP en fonction des contextes et des populations à l'échelle locale tout en assurant la fidélité aux éléments de modèle du NFP.

2. Assurer la faisabilité et l'acceptabilité du programme adapté dans une étude pilote de faible envergure.

3. Évaluer le programme adapté dans un ECR à grande échelle.

4. Diffuser le programme adapté dans la société visée si l'évaluation révèle des résultats positifs significatifs.

Abréviations : ECR, essai contrôlé randomisé; NFP, Nurse-Family Partnership. 
TABLEAU 2

Chronologie des activités liées à l'adaptation, à la mise à l'épreuve et à l'évaluation du Nurse-Family Partnership au Canada

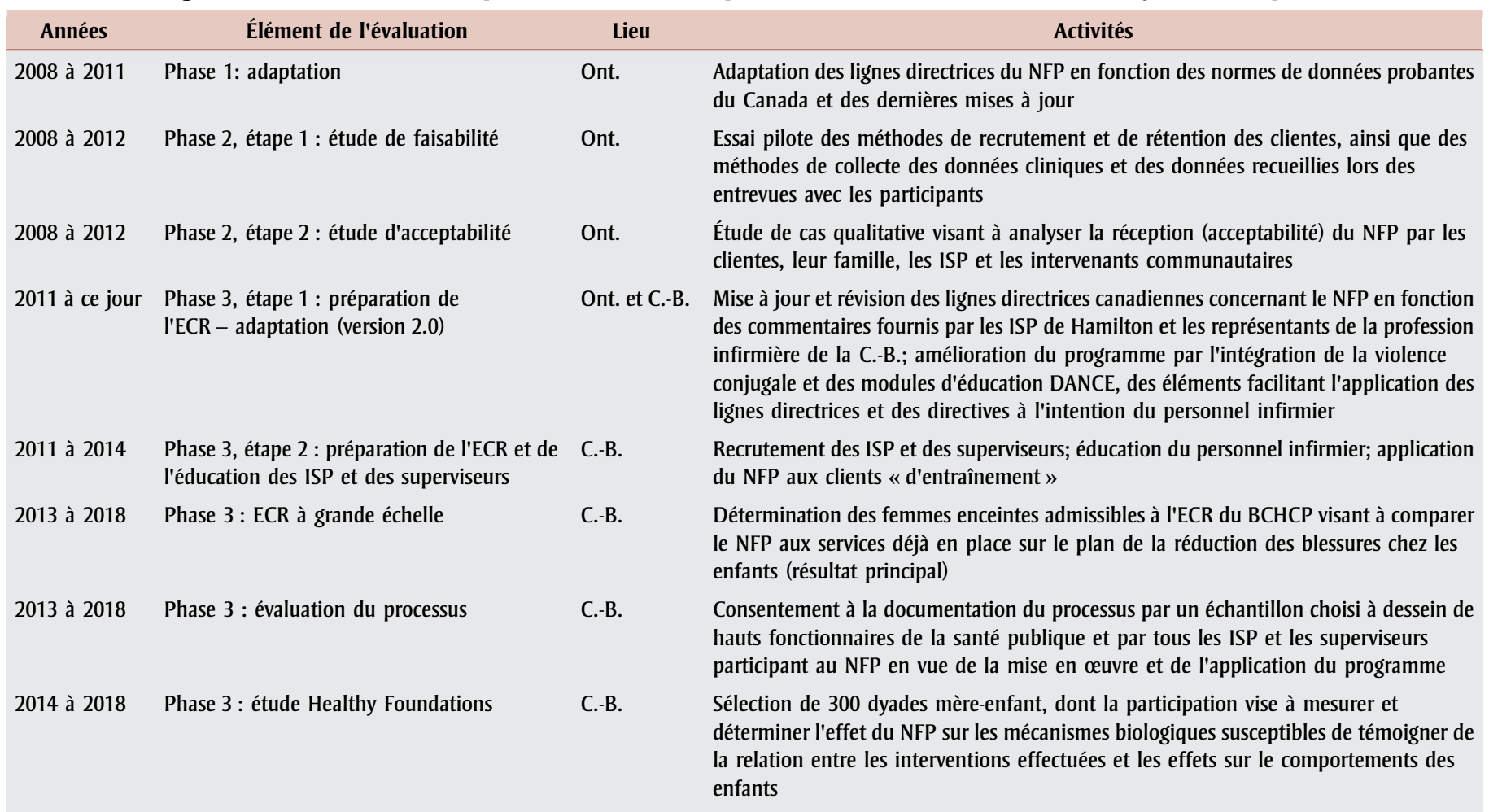

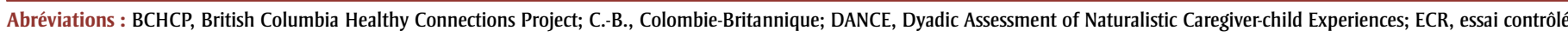
randomisé; ISP, infirmières et infirmiers en santé publique; NFP, Nurse-Family Partnership; Ont., Ontario.

\section{Résultats}

\section{Adaptation du Nurse-Family Partnership en vue de son utilisation au Canada}

La première étape stratégique de l'introduction du NFP au Canada a été l'établissement d'un partenariat entre les chercheurs de l'Université McMaster à Hamilton (Ontario) et les principaux responsables des Services de santé publique de la ville. Cette ville était le lieu idéal pour un essai pilote du NFP compte tenu du grand appui de la collectivité à l'endroit du programme, de la présence d'une unité de santé publique offrant des programmes fondés sur des données probantes solides et du partenariat en cours avec un groupe de recherche de l'Université McMaster ayant des connaissances spécialisées sur les évaluations d'interventions en matière de maltraitance envers les enfants et de violence conjugale. Il a été déterminé que le déploiement du NFP serait assuré conjointement par les organismes de santé publique et les infirmières et infirmiers en santé publique (ISP), car les ISP ont déjà l'habitude de faire des visites à domicile auprès des familles vulnérables durant la période périnatale.
Dans la plupart des provinces et des territoires du Canada, les ISP détiennent un baccalauréat en soins infirmiers, respectant du même coup l'un des éléments du modèle d'intervention du NFP. Il a fallu nommer un responsable de programme à l'échelle de l'unité de santé publique locale pour permettre la réaffectation d'une partie du financement provincial accordé aux visites à domicile vers le lancement et la mise à l'épreuve de ce programme ${ }^{12}$.

L'adaptation canadienne de la documentation du NFP - les lignes directrices relatives aux visites (recommandations destinées à orienter chacune des visites), les directives à l'intention du personnel infirmier, les feuilles de travail sur les clients et les autres ressources nécessaires durant les visites à domicile - se fait dans le cadre d'un projet de collaboration entre les responsables de la gestion de la santé publique, les ISP de première ligne - qui fournissent des comptes rendus relatant leurs expériences cliniques avec le NFP - et les chercheurs en soins infirmiers (SJ, DS) qui intègrent les connaissances sur la pratique clinique du personnel infirmier avec les pratiques exemplaires et les données de recherche existantes. À ce jour, la documentation américaine a été adaptée sur les points suivants :

1) intégration des normes de pratique et des lignes directrices sur les pratiques exemplaires du Canada concernant des sujets comme les calendriers d'immunisation, la consommation d'aliments et de nutriments et la prévention des blessures,

2) amélioration de la documentation pour répondre à des besoins ou à des priorités établis à l'échelle locale, par exemple pour se conformer aux recommandations en faveur de l'allaitement de l'Initiative Amis des bébés ${ }^{13}$,

3) intégration des innovations du NFP, notamment une intervention pour cerner les cas de violence conjugale et y répondre ${ }^{14}$,

4) mise au point d'un outil pour observer les interactions parent-enfant, en l'occurrence le Dyadic Assessment of Naturalistic Caregiver-child Experiences (DANCE) ${ }^{15}$.

Il est conseillé aux ISP travaillant à l'élaboration de lignes directrices destinées 
aux provinces d'utiliser toute ligne directrice locale ou provinciale qui diffère des lignes directrices canadiennes. À l'étape de l'adaptation, une grande attention a été accordée au respect des exigences en matière de présentation, à savoir :

1) conformité avec la Loi de 2005 sur l'accessibilité pour les personnes handicapées de l'Ontario ${ }^{16}$,

2) conversion des unités impériales en unités métriques,

3) intégration ou substitution de diagrammes pour renforcer les principes de l'Initiative Amis des bébés ou prendre en compte la diversité des clients canadiens,

4) inclusion dans chaque document d'une indication écrite que les adaptations ont été réalisées avec l'autorisation du Bureau national des services du NFP.

Avant d'être utilisés sur le terrain, tous les documents adaptés ont été soumis au Centre de recherche sur la prévention de l'Université du Colorado à Denver pour examen, commentaires et approbation.

\section{Évaluation de la faisabilité et de l'acceptabilité du NFP au Canada}

L'étude de faisabilité visait à évaluer 1) si les femmes enceintes répondant aux critères d'admissibilité au NFP pouvaient être orientées vers le programme et bénéficier de celuici, 2) si le programme pouvait être offert par un organisme de santé publique d'une manière fidèle aux 18 éléments de modèle du $\mathrm{NFP}^{17}$, 3) si des données sur le programme pouvaient être colligées par l'organisme responsable de la mise en œuvre et 4) si les données sur les résultats chez les clientes pouvaient être collectées par le groupe de recherche. Pour atteindre ces objectifs, Jack et ses collaborateurs ont réalisé de 2008 à 2012 une étude pilote à Hamilton faisant appel à des méthodes mixtes ${ }^{18}$. Au cours de cette période, ils ont vérifié l'admissibilité au NFP de 424 femmes orientées vers des soins prénataux à l'Unité de santé publique de Hamilton. Les critères d'admissibilité étaient les suivants : être jeune (21 ans ou moins), avoir un faible revenu, être orientée avant la fin de la $28^{\mathrm{e}}$ semaine de la grossesse et être en voie d'accoucher pour la première fois. Au total,
135 femmes enceintes ont été jugées admissibles au NFP (32\% de l'ensemble des orientations vers les soins prénataux) et 108 (80\%) d'entre elles ont consenti à participer à l'étude pilote. De plus amples détails sur la méthodologie de l'étude pilote sont fournis ailleurs ${ }^{18}$.

Cinq des 108 participantes ont refusé tout contact ultérieur avec le groupe de recherche et l'une a été perdue de vue avant l'entrevue initiale. Des 102 femmes restantes, 71 ont terminé l'entrevue finale au moment des 2 ans de leur enfant. La plupart (87\%) des femmes se sont jointes au programme lors de la $25^{\mathrm{e}}$ semaine de leur grossesse ou plus tôt (la norme internationale est de $60 \%$, ce qui correspond à 16 semaines de grossesse) et $77 \%$ des participantes étaient âgées de 16 à 19 ans.

Les résultats de l'étude pilote ont montré qu'il était faisable :

- d'offrir le programme NFP par l'entremise des unités de santé publique,

- d'assurer la prestation de l'intervention par les ISP,

- de recevoir des orientations appropriées des partenaires communautaires,

- de recruter les participantes admissibles,

- de visiter à domicile la population cible constituée des jeunes nouvelles mères à faible revenu.

Des données détaillées sur le programme NFP ont aussi été tirées des formulaires d'évaluation du personnel infirmier dans lesquels sont notés systématiquement l'état de santé et les comportements liés à la santé de chaque mère, l'état de santé et le développement de l'enfant ainsi que le recours à d'autres services de santé et services communautaires. Un processus permettant au groupe de recherche de collecter des données sur les résultats chez les clients a aussi été mis en œuvre.

Outre l'évaluation de la faisabilité de la prestation du programme, Jack et ses collaborateurs ont aussi mené une étude de cas qualitative sur la réception du NFP par les familles de Hamilton ${ }^{18}$. Dans les recherches sur les interventions, les études d'acceptabilité constituent un important complément aux études de faisabilité classiques. La détermination de l'acceptabilité d'une intervention dans un nouveau contexte comporte deux avantages : 1) elle permet aux principales parties concernées de s'impliquer tôt dans le processus, ce qui peut faciliter le déploiement ultérieur de l'intervention et 2) elle fait ressortir les éléments de l'intervention qui pourraient devoir être davantage adaptés pour répondre aux besoins du public cible, des fournisseurs de soins et de la collectivité locale ${ }^{19}$. Une étude d'acceptabilité consiste essentiellement en un examen du contexte local destiné à définir si et comment l'intervention sera réalisée et reçue par le public cible. Elle permet aussi de déterminer si l'intervention correspond aux besoins du public cible et de la collectivité.

Avant d'ajouter une nouvelle intervention de santé publique au programme provincial de visites à domicile, il était important de vérifier la bonne réception du NFP par la collectivité ainsi que le degré de préparation au NFP. Le NFP différait considérablement du programme déjà en place de visites à domicile de l'Ontario sur quatre points : il ciblait les nouvelles mères défavorisées sur le plan socioéconomique, il était offert par des infirmières et infirmiers autorisés titulaires d'un baccalauréat et il prévoyait un nombre accru de visites à domicile, ces visites commençant plus tôt dans la grossesse.

À Hamilton, les mères défavorisées sur le plan socioéconomique ayant bénéficié des visites à domicile ont fait une réception positive au NFP comme intervention de santé publique précoce ${ }^{20}$. À l'échelle organisationnelle, cette intervention a créé un environnement où les ISP pouvaient administrer des soins infirmiers globaux et mettre en pratique toutes les compétences de leur champ d'exercice, établir des relations thérapeutiques profondes avec les clients et compter sur l'infrastructure, les ressources et le matériel nécessaires pour évaluer et aider les clientes confrontés à des problèmes de santé et à des problèmes sociaux complexes (troubles de santé mentale, toxicomanies, exposition à la violence, etc. ${ }^{20}$. Les professionnels de la collectivité qui étaient responsables de l'orientation des femmes vers le programme ont déclaré que le NFP venait combler une lacune importante dans la prestation de services de santé aux familles les plus vulnérables ${ }^{18}$. 


\section{Éléments du modèle canadien du Nurse- Family Partnership}

Le modèle canadien du NFP est fondé sur des constatations tirées de l'étude de cas de Jack et de ses collaborateurs ${ }^{18}$ de même que sur les exigences du Centre de recherche sur la prévention de l'Université du Colorado à Denver et du Bureau national des services du NFP. Les résultats de l'étude de cas menée à Hamilton ont orienté :

1) l'élaboration des lignes directrices canadiennes relatives aux visites, en particulier les directives et les formulaires utilisés pour documenter les pratiques infirmières,

2) la recommandation en faveur de la prestation du NFP par les organismes de santé publique et les ISP,

3) les recommandations en faveur de l'embauche et de la dotation, qui concernent aussi la charge de travail,

4) l'aspect progressif de la mise en œuvre, c'est-à-dire l'attribution initiale aux infirmières et infirmiers d'un petit nombre de clients «d'entraînement » avec lesquels travailler à mesure qu'ils suivent les formations exigées par le NFP.

Le tableau 3 contient un résumé des éléments du modèle canadien du NFP.

\section{Préparation en vue de l'évaluation à grande} échelle du NFP au Canada

La préparation et la réalisation d'une étude à grande échelle visant à évaluer l'efficacité du NFP au Canada nécessitent un niveau important de financement, d'expertise de recherche et d'engagement communautaire.

TABLEAU 3

Éléments du modèle canadien du Nurse-Family Partnership

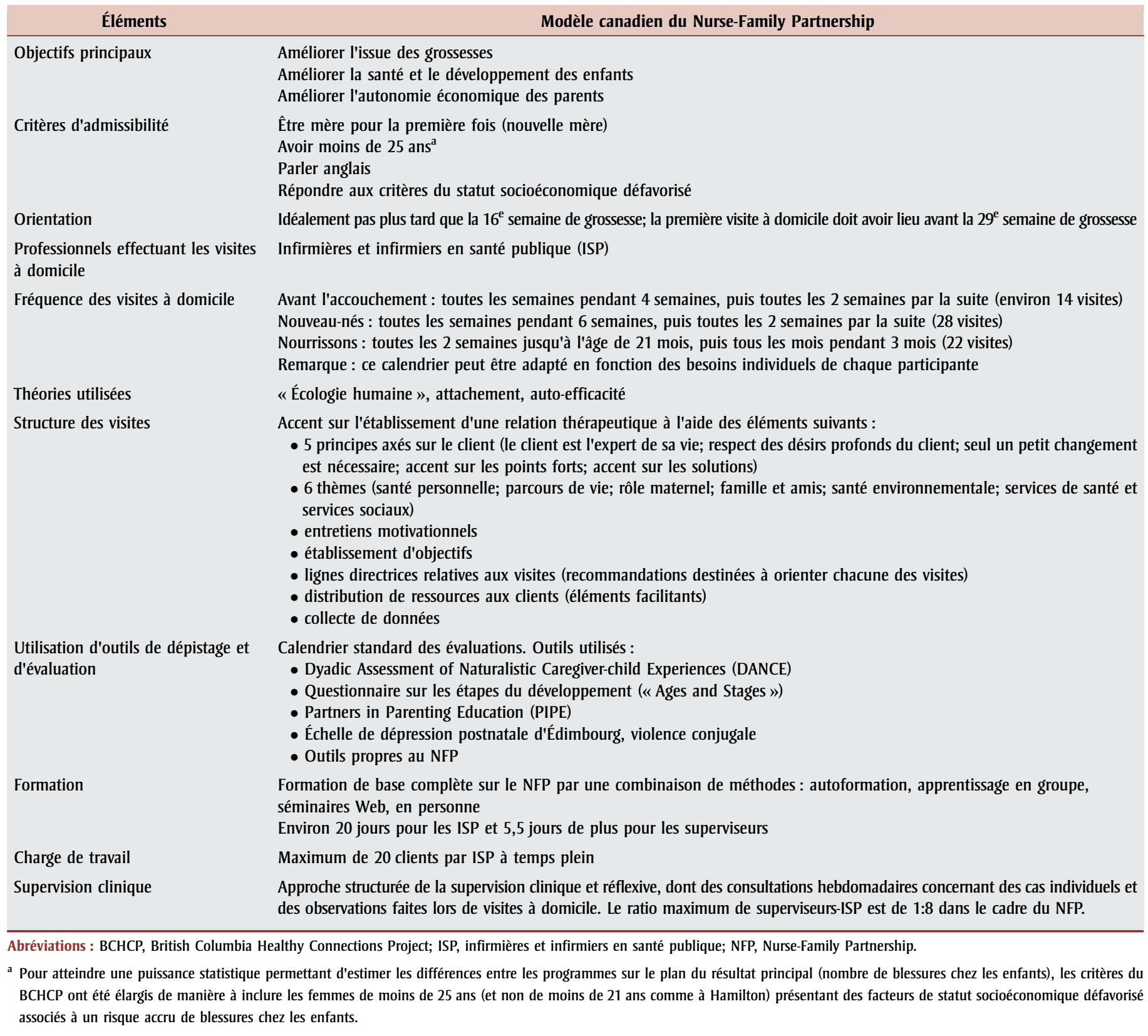


Les efforts déployés pour étendre l'étude menée à Hamilton au reste de l'Ontario n'ont pas porté fruit ${ }^{12}$. En 2010, la ColombieBritannique a publié Healthy Minds, Healthy People: A Ten-Year Plan to Address Mental Health and Substance Use $e^{21}$, un plan préconisant une intervention axée sur des visites au domicile des nouvelles mères défavorisées et de leurs enfants par des infirmières et infirmiers. Les visites au domicile des femmes enceintes les plus vulnérables par du personnel infirmier sont aussi citées comme une approche prometteuse pour améliorer la capacité des systèmes de santé dans le document B.C.'s Guiding Framework for Public Health ${ }^{22}$.

Dans le cadre de leur plan de dix ans sur la santé mentale ${ }^{21}$, le ministère de la Santé et le ministère du Développement des enfants et de la Famille de la Colombie-Britannique ont conjointement invité le Centre des politiques sur la santé des enfants de la Faculté des sciences de la santé de l'Université Simon Fraser à explorer comment évaluer le NFP en Colombie-Britannique en collaboration avec les responsables de l'étude pilote sur le NFP de l'Université McMaster. Les deux ministères ont aussi mis sur pied un comité consultatif provincial constitué de hauts fonctionnaires des autorités sanitaires de la ColombieBritannique, des secteurs de prestation de services du ministère du Développement des enfants et de la Famille, des organisations autochtones et d'autres organismes tels que l'Agence de la santé publique du Canada. Les réunions de ce comité ont permis d'échanger de l'information sur le NFP et, dans le cas des ministères de la Santé et du Développement des enfants et de la Famille, de dégager des consensus provinciaux quant aux modalités de l'évaluation du NFP.

Le B.C. Healthy Connections Project (BCHCP), qui bénéficie du financement du ministère de la Santé et de l'appui du ministère du Développement des enfants et de la Famille, a été lancé officiellement en 2012 en étroite collaboration avec cinq autorités sanitaires participantes (Fraser Health, Vancouver Coastal Health, Interior Health, Northern Health et Island Health). Le $\mathrm{BCHCP}$ réunit maintenant trois études primaires: un ECR visant à évaluer l'impact du NFP sur les résultats de santé et les résultats sociaux (notamment la santé mentale et le développement durant la petite enfance et le parcours de vie de la mère), une évaluation du processus visant à décrire la mise en œuvre et la prestation du NFP et enfin l'étude Healthy Foundations, qui portait sur l'impact du NFP sur la physiologie et le fonctionnement des nourrissons. Ces études ont débuté en octobre 2013 et devraient se poursuivre jusqu'en 2016. La collecte de données continuera jusqu'à ce que les enfants participants atteignent l'âge de 2 ans.

\section{Essai contrôlé randomisé du BCHCP : évaluation de l'efficacité du NFP}

Le BCHCP comprend un ECR visant à évaluer l'efficacité du NFP dans cinq sphères: grossesse, naissance, santé et développement durant la petite enfance et enfin parcours de vie de la mère. L'impact concret du NFP sur les mères et les enfants ainsi que les facteurs de risque et de protection connexes seront évalués à l'égard de ces cinq sphères. Le NFP sera comparé aux services de santé et aux services sociaux déjà offerts en Colombie-Britannique (« services déjà en place ») du début de la grossesse jusqu'aux 2 ans de l'enfant. Comme le préconise un rapport récent sur l'utilisation des ECR dans l'élaboration des politiques publiques, la réalisation d'un essai pour déterminer l'efficacité du NFP en Colombie-Britannique devra garantir que le groupe bénéficiant du NFP et le groupe bénéficiant des services déjà en place seront appariés aussi étroitement que possible ${ }^{23}$. L'évaluation du NFP à l'aide d'un ECR permet de mieux contrôler les facteurs externes qui pourraient influer sur les observations. La méthodologie de l'ECR du $\mathrm{BCHCP}$ est brièvement décrite plus loin.

Les femmes admissibles à l'ECR du BCHCP sont de nouvelles mères défavorisées sur le plan socioéconomique (p. ex. à faible niveau de scolarité, à faible revenu, monoparentales) âgées de moins de 25 ans. La taille de la population souhaitée est de 1000 femmes, qui seront réparties aléatoirement entre un groupe bénéficiant du NFP et un groupe bénéficiant des services déjà en place. Les données seront collectées au départ, lors d'entrevues et par l'établissement de liens avec des données administratives sur la santé, puis à des intervalles réguliers jusqu'au deuxième anniversaire de l'enfant. Les femmes classées dans le groupe
« d'intervention » bénéficieront du NFP et pourront recevoir des services déjà en place dans leur autorité sanitaire alors que les femmes du groupe «de comparaison» seront limitées aux services déjà en place. Les services déjà en place, qui varient d'un endroit à l'autre dans la province, sont constitués des soins de santé primaires standard, des programmes de santé publique (cours prénataux, programmes de soutien à la grossesse et visites à domicile par des infirmières et infirmiers ne participant pas au NFP) et une variété de programmes généraux et ciblés d'éducation parentale et de développement sain des jeunes enfants.

Le BCHCP produira aussi des données détaillées permettant d'évaluer les services déjà en place en Colombie-Britannique. L'utilisation des services sera mesurée au sein d'un large éventail de secteurs de la santé et de secteurs sociaux durant les deux premières années de vie de l'enfant: soins de santé primaires dispensés aux mères et aux enfants, programmes de soins infirmiers périnataux standard, programmes d'éducation des parents, programmes de développement sain des jeunes enfants, visites de l'enfant aux urgences, hospitalisations de l'enfant, enquêtes des services de protection de l'enfance, placements dans une famille d'accueil, démêlés de la mère avec le système de justice pénale, soutien au revenu familial, etc. Les données relatives à l'utilisation des services seront aussi collectées lors des entrevues menées dans le cadre du BCHCP et par l'établissement de liens avec des données administratives sur la santé. Certaines données détaillées sur le programme NFP seront tirées des formulaires d'évaluation du personnel infirmier. Les articles à venir offriront une analyse approfondie du recours aux services déjà en place et le NFP en Colombie-Britannique.

À la lumière des données issues de l'ECR réalisé aux États-Unis, nous avons avancé l'hypothèse que le résultat principal du NFP sera la réduction du nombre de blessures des enfants entre la naissance et l'âge de 2 ans. Les résultats secondaires seront:

- la réduction de la consommation d'alcool et de drogues par la mère durant la grossesse,

- l'amélioration du développement langagier et cognitif de l'enfant à l'âge de 2 ans, 
- l'amélioration du comportement de l'enfant à l'âge de 2 ans,

- l'amélioration du parcours de vie de la mère 2 ans après l'accouchement (p. ex. nombre de grossesses subséquentes).

Nous évaluerons également les facteurs de risque et de protection connexes (p. ex. santé physique de l'enfant, santé mentale de la mère, rôle parental, statut socioéconomique, exposition à de la violence conjugale). Nous préparons actuellement une évaluation de l'impact à long terme du NFP tout au long de l'enfance ainsi que pendant l'adolescence. Les données sur le recours aux services permettront de réaliser une évaluation économique. Pour faire état de l'utilisation des services d'un point de vue sociétal plus global, nous recueillerons aussi des données sur les services non utilisés et les motifs de cette absence d'utilisation. Des responsables de l'élaboration des politiques participeront à chaque étape de l'évaluation. Nous avons pour objectif commun d'améliorer la vie des enfants de la Colombie-Britannique dès leur plus jeune âge.

\section{Évaluation du processus dans le cadre du BCHCP}

Le NFP est offert dans cinq autorités sanitaires de la Colombie-Britannique, chacune différente par sa géographie, par les caractéristiques de sa population et par la culture des partenariats et des collaborations mis à contribution pour appuyer le NFP à l'échelle communautaire. Ce degré de complexité pose des difficultés pour l'évaluation de l'efficacité des interventions et de l'influence potentielle des mécanismes causaux des interventions sur les résultats escomptés ${ }^{24}$. Outre leur utilisation pour mesurer les résultats d'essais, les évaluations de processus sont de plus en plus combinées avec des ECR pour analyser en profondeur des interventions complexes. Cette évaluation du processus aidera à définir les facteurs favorables et défavorables au succès du NFP dans un large éventail de collectivités. Les chercheurs exploreront aussi des méthodes aptes à surmonter les obstacles recensés à la mise en œuvre et à la prestation du programme.

L'évaluation du processus dans le cadre du $\mathrm{BCHCP}$ vise avant tout à déterminer et à comparer, dans les cinq autorités sanitaires participantes de la Colombie-Britannique :

- comment le NFP est mis en œuvre,

- si l'intervention est fidèle aux éléments de modèle du NFP,

- la dose de l'intervention fournie et reçue,

- les problèmes de recrutement des clientes et de maintien en poste des ISP,

- les facteurs contextuels au niveau de la cliente, du fournisseur de soins, de l'organisme et de la collectivité qui influent sur la mise en œuvre et la prestation (dont une comparaison entre les contextes urbains et ruraux),

- comment les ISP définissent et règlent les problèmes liés à la prise en charge par les services d'aide à l'enfance, à la violence conjugale et aux troubles de santé mentale (incluant les toxicomanies).

Les chercheurs feront l'évaluation du processus selon un plan de recherche utilisant des méthodes mixtes convergentes et parallèles ${ }^{25}$.

Tous les infirmières et infirmiers de la Colombie-Britannique et leurs superviseurs participant à la prestation du NFP dans l'exercice de leurs responsabilités de santé publique seront invités à participer à l'étude. Dans chaque autorité sanitaire, on choisira un échantillon de 10 à 15 gestionnaires et directeurs de la santé publique responsables du BCHCP ou du NFP. Des données seront collectées à intervalles réguliers entre 2013 et 2018 pour documenter le processus de mise en œuvre dans chaque autorité sanitaire. Tous les infirmières et infirmiers et les superviseurs, de même que le coordonnateur provincial du NFP, prendront part à une entrevue en profondeur (ou à un groupe de discussion) tous les six mois et les gestionnaires de la santé publique participeront à une entrevue détaillée tous les ans. Les superviseurs devront remplir tous les mois les documents requis (notamment les sommaires des rencontres de supervision réflexives entre les superviseurs et les ISP, des réunions d'équipe et des conférences de cas) et les données seront rassemblées tous les 6 mois. Les données sur la fidélité au NFP fournies par le ministère de la Santé seront recueillies tous les 3 mois. La collecte des données quantitatives et qualitatives permettra de combiner les points forts des deux méthodes de recherche et de comparer, corroborer et expliquer les résultats et les écarts observés dans les cinq autorités sanitaires.

\section{Étude Healthy Foundations}

Nous souhaitions, en plus d'évaluer l'efficacité du NFP et le déroulement de sa mise en œuvre, examiner les mécanismes biologiques susceptibles de témoigner de la relation entre les interventions effectuées et les effets sur le comportement des enfants. Il a été démontré que les expériences négatives durant l'enfance comme la violence et la pauvreté ont des conséquences durables sur la santé cognitive, mentale et physique ${ }^{26,27}$. Des axes de recherche parallèles chez l'humain et l'animal ont montré que des conditions de vie difficiles en bas âge perturbent de façon durable l'organisme, notamment en altérant le système de réponse au stress (axe hypothalamo-hypophyso-surrénalien) ${ }^{28,29}$ et le fonctionnement du système immunitaire $^{30,31}$, ce qui affecte le développement et la santé du cerveau. De nouvelles données indiquent que ces expériences peuvent laisser, au niveau moléculaire et génomique, une « empreinte biologique » qui détermine la vulnérabilité plus tard au cours de la vie ${ }^{27}$. Ces expériences mènent à des trajectoires développementales qui sont difficiles à modifier ultérieurement ${ }^{32}$. À notre connaissance, aucune étude longitudinale n'a encore porté sur l'incidence des interventions préventives sur les caractéristiques biologiques des mères et de leurs enfants et aucune étude n'a examiné si ces interventions pouvaient désamorcer le conditionnement biologique des maladies.

En juillet 2013, nous avons reçu du financement pour intégrer au BCHCP une sous-étude, l'étude Healthy Foundations, comprenant 300 dyades (150 personnes dans le groupe d'intervention et 150 dans le groupe de comparaison) et visant trois objectifs principaux: 1) déterminer si le NFP a un effet sur la physiologie des nourrissons, mesuré par le fonctionnement de l'axe hypothalamo-hypophysosurrénalien et les marqueurs épigénétiques, 2) examiner si le NFP a un impact sur les taux de cortisol pendant la grossesse et si ceux-ci sont associés à des 
altérations de la physiologie du stress, de la fonction immunitaire et de la méthylation de l'ADN chez le nourrisson et 3) examiner si les altérations des marqueurs biologiques permettent d'expliquer l'impact du NFP sur les résultats liés au développement de l'enfant à 2 ans. Nous prélèverons des échantillons biologiques auprès des sujets des deux groupes d'étude (NFP et services déjà en place), des mères (cheveux) comme des nourrissons (salive) pour mesurer les niveaux de stress et la fonction immunitaire. Des prélèvements buccaux seront faits sur les nourrissons en vue d'examiner les marqueurs influant sur l'expression génique. Une meilleure compréhension des aspects biologiques des expériences négatives en bas âge et des moyens d'annuler leurs répercussions négatives (à l'aide du NFP) fournira un cadre solide qui orientera la recherche fondamentale et appliquée, les pratiques et les politiques.

\section{Analyse}

Le NFP est un programme très prometteur pour influencer les déterminants de la santé des nourrissons - comportements prénataux, rôle parental et violence - qui ont une incidence sur la santé mentale et l'apprentissage durant la petite enfance et plus tard au cours de la vie. Bien que de nombreux organismes de santé publique soient prêts à mettre en œuvre le NFP, cette intervention de santé publique n'a jamais été mise à l'épreuve au Canada. On ne sait donc pas s'il est possible de reproduire - et approfondir - les résultats des essais menés aux États-Unis.

Le recours à une approche systématique pour adapter la documentation américaine du NFP et étudier la faisabilité et l'acceptabilité de cette intervention de santé publique a ouvert la voie à une évaluation de son efficacité. Le BCHCP est prépare l'évaluation et l'adaptation du NFP en vue de son utilisation à long terme en Colombie-Britannique. Ces travaux pourraient être utilisés pour mettre en œuvre le NFP ailleurs au Canada. Les conclusions de l'évaluation du processus orienteront les adaptations ultérieures des lignes directrices et de l'éducation de base du personnel infirmier sur le NFP au Canada.
Le BCHCP contribuera aussi à enrichir le corpus de données du NFP par l'inclusion des données de l'étude Healthy Foundations. Les observations de l'évaluation du processus vont influencer notre compréhension des mesures à prendre pour adapter le modèle de prestation de services en fonction des besoins des familles vivant dans des collectivités rurales et éloignées. Cela dit, l'élément le plus caractéristique du BCHCP est qu'il est déjà intégré dans le système de santé de la Colombie-Britannique, sous forme de collaboration étroite avec les responsables des politiques touchant la santé des enfants du ministère de la Santé, du ministère du Développement des enfants et de la Famille et de cinq autorités sanitaires. Il permet du même coup d'évaluer l'efficacité du NFP en situation réelle et d'influencer positivement les soins reçus par les mères et leurs enfants. Par exemple, les partenaires stratégiques du BCHCP se sont déjà engagés à adopter le NFP comme programme permanent de soins de santé maternelle et infantile en Colombie-Britannique si l'ECR s'avère positif.

\section{Conclusion}

En 2004, le Centre de recherche sur la prévention de l'Université du Colorado à Denver dirigé par le $\mathrm{D}^{\mathrm{r}}$ Olds a commencé à répondre aux demandes des chercheurs et des responsables des politiques hors ÉtatsUnis qui étaient intéressés à mettre en œuvre le NFP dans leur pays. Le Centre de recherche sur la prévention a établi un processus en quatre étapes (tableau 1) pour aider les partenaires étrangers à adapter et à mettre à l'épreuve le NFP dans leur société tout en assurant la fidélité aux éléments fondamentaux du modèle du programme. À la lumière des derniers développements dans la recherche sur la prévention, nous recommandons au Centre de recherche d'ajouter aux obligations des sites étrangers la tenue d'une évaluation du processus menée conjointement avec l'ECR déjà exigé.

À l'heure actuelle, tous les ECR effectués hors des États-Unis le sont de façon indépendante. Il n'existe pas de mécanisme officiel qui permettrait aux chercheurs internationaux sur le NFP de collaborer ou de se consulter. Étant donné la variété des contextes dans lesquels se fait l'évaluation de cette intervention de santé publique, il est impératif d'établir un mécanisme de collaboration internationale sur divers éléments du programme. Des mesures ont maintenant été prises pour permettre aux pays travaillant à la mise en œuvre du NFP de se réunir et de résoudre leurs problèmes communs, notamment la création d'un comité consultatif clinique et d'un réseau de chefs de file cliniques. Récemment, un petit groupe international a été formé pour garantir la qualité de la mise en œuvre du NFP dans de nouveaux pays et sociétés. En octobre 2014, la première réunion des représentants (responsables de l'éducation et de la mise en œuvre) de sept sociétés faisant des travaux sur le NFP (Australie, Angleterre, Canada, Écosse, États-Unis, Irlande du Nord, Pays-Bas) a eu lieu à Denver. Cette réunion visait à examiner et à approfondir le NFP à propos des principes et des théories concernant l'éducation, des compétences infirmières ainsi que du contenu et des processus essentiels (ou facultatifs) destinés à orienter et favoriser la mise sur pied d'un réseau d'éducation sur le NFP. Une séance préliminaire a porté sur le processus d'élaboration et de mise en œuvre des innovations comme le DANCE et sur une intervention ayant pour but de réduire la violence conjugale. Ces deux éléments font partie de l'intervention de base dans le cadre du NFP à l'étude en Colombie-Britannique. On espère et on croit que, au fil de l'avancement de la mise en œuvre du NFP à l'échelle mondiale, les chercheurs sur le NFP de partout dans le monde uniront leurs efforts pour établir un réseau de collaboration.

En Colombie-Britannique, nous sommes en train de préparer le suivi de notre échantillon de 1000 enfants pendant 10 ans ou plus, soit jusqu'au milieu de l'enfance et à l'adolescence. Cela nous permettra d'évaluer en profondeur l'impact potentiel du NFP sur plusieurs résultats de santé chez les enfants et résultats de santé publique : la prévention des troubles de santé mentale les plus courants (notamment l'anxiété, la dépression, les troubles du comportement et les toxicomanies), l'amélioration des résultats scolaires et la réduction de la violence et de ses conséquences. Le suivi à long terme ouvre aussi la voie à une première 
évaluation économique détaillée du NFP au Canada. Enfin, et peut-être surtout, nous souhaitons utiliser le BCHCP pour soutenir et diffuser l'approche axée sur l'enfant qui a été adoptée par le système de santé publique de la Colombie-Britannique, de manière à l'étendre aux autres provinces et à collaborer avec les partenaires internationaux qui ont les mêmes objectifs que nous en ce qui concerne la santé des enfants.

\section{Remerciements}

Les membres du groupe scientifique du BCHCP sont : Ronald G. Barr, M.A. MDCM, FRCPC (Université de la Colombie-Britannique); Michael Boyle, Ph. D. (Université McMaster); Amiram Gafni, D. Sc. (Université McMaster); Kaitlyn Hougham, M. Sc. (Université Simon Fraser); Lenora Marcellus, IA, Ph. D. (Université de Victoria); Lawrence McCandless, Ph. D. (Université Simon Fraser); Cody Shepherd, B.A. (Hon.) (Université Simon Fraser); Lil Tonmyr, Ph. D. (Agence de la santé publique du Canada); Natasha Van Borek, M. Sc. SPP (Université McMaster) et Colleen Varcoe, IA, Ph. D. (Université de la ColombieBritannique). L'Agence de la santé publique du Canada a accordé des fonds pour l'adaptation de la documentation du NFP. Les Services de santé publique de la Ville de Hamilton ont financé les études de faisabilité et d'acceptabilité en collaboration avec deux organismes de protection de l'enfance (la Société catholique d'aide à l'enfance de Hamilton et la Société d'aide à l'enfance de Hamilton), la Fondation communautaire de Hamilton, l'Hôpital pour enfants McMaster, le Secrétariat des soins infirmiers du ministère de la Santé et des Soins de longue durée de l'Ontario ainsi que le Centre d'excellence de l'Ontario en santé mentale des enfants et des adolescents. Le BCHCP est financé par le ministère de la Santé de la Colombie-Britannique avec l'aide du ministère du Développement des enfants et de la Famille et des autorités sanitaires Fraser Health, Interior Health, Island Health, Northern Health et Vancouver Coastal Health. D’autres fonds ont été fournis par la Fondation Djavad Mowafaghian. L'évaluation du processus menée dans le cadre du BCHCP a bénéficié d'un financement additionnel de la Section des blessures et de la violence envers les enfants du Centre de prévention des maladies chroniques de l'Agence de la santé publique du Canada. Létude Healthy Foundations a quant à elle été financée par les Instituts de recherche en santé du Canada. Harriet MacMillan est appuyée par la Chaire Chedoke de recherche en psychiatrie. Charlotte Waddell est appuyée par la Chaire de recherche du Canada sur la politique en matière de santé des enfants.

\section{Références}

1. Afifi TO, MacMillan HL, Boyle M, Taillieu T, Cheung K, Sareen J. Child abuse and mental disorders in Canada. CMAJ. 2014;186(9): E324-E332.

2. Duggan AK, McFarlane EC, Windham AM et collab. Evaluation of Hawaii's Healthy Start program. Future Child. 1999;9(1):66-90.

3. MacMillan HL, Wathen $\mathrm{CN}$, Barlow J, Fergusson DM, Leventhal JM, Taussig HN. Interventions to prevent child maltreatment and associated impairment. Lancet. 2009;373: 250-266.

4. Olds DL, Sadler S, Kitzman H. Programs for parents of infants and toddlers: recent evidence from randomized trials. J Child Psych Psychiatry. 2007;48:355-391.

5. Olds DL, Kitzman H, Knudtson MD, Anson E, Smith JA, Cole R. Effect of home visiting by nurses on maternal and child mortality: results of a 2-decade follow-up of a randomized clinical trial. JAMA Pediatr. 2014;168(9):800-806.

6. Aos S, Lieb R, Mayfield J, Miller M, Pennucci A. Benefits and costs of prevention and early intervention programs for youth. Olympia (WA): Washington State Institute for Public Policy; 2004.

7. Olds DL, Hill PL, O’Brien R, Racine D, Moritz P. Taking preventive intervention to scale: The Nurse-Family Partnership. Cogn Behav Pract. 2003;10:278-290.

8. Prevention Research Center for Family and Child Health. Nurse-Family Partnership international program [Internet]. Denver (CO): University of Colorado Denver; [consultation le 4 décembre 2014]. Consultable en ligne à la page http://www. ucdenver.edu/academics/colleges/medicalschool/departments/pediatrics/research/ programs/prc/research/international/Pages/ international.aspx
9. Barnes J. From evidence-base to practice: implementation of the Nurse Family Partnership programme in England. J Child Serv. 2010;5:4-17

10. Mejdoubi J, van den Heijkant S, Struijf E, van Leerdam F, HiraSing R, Crijnen A. Addressing risk factors for child abuse among high risk pregnant women: design of a randomised controlled trial of the nurse family partnership in Dutch preventive health care. BMC Public Health. 2011;11:823.

11. Mejdoubi J, van den Heijkant S, van Leerdam FJ, Heymans MW, Hirasing RA, Crijnen AA. Effect of nurse home visits vs usual care on reducing intimate partner violence in young high-risk pregnant women: a randomized controlled trial. PLoS ONE. 2013; 8(10):e78185.

12. Jack SM, MacMillan HL. Adaptation and evaluation of the Nurse-Family Partnership in Canada [Internet]: Early Childhood Matters. 2014 [consultation le 24 novembre 2014]: 43-46. Consultable en ligne à la page http:// earlychildhoodmagazine.org/adaptation-andevaluation-of-the-nurse-family-partnership-incanada/

13. Pound CM, Unger SL. L'Initiative Amis des bébés : protéger, promouvoir et soutenir l'allaitement. Paediatr Child Health. 2012; 17(6):322-326.

14. Jack SM, Ford-Gilboe M, Wathen $\mathrm{CN}$, et al. Development of a nurse home visitation intervention for intimate partner violence. BMC Health Serv Res. 2012;12:50.

15. Olds D, Donelan-McCall N, O’Brien R, et al. Improving the Nurse-Family Partnership in community practice. Pediatrics. 2013;132: S110-S117.

16. Ministère des Services sociaux et communautaires de l'Ontario. Loi sur l'accessibilité pour les personnes handicapées de l'Ontario [Internet]. Toronto (Ont.) : Ministère des Services sociaux et communautaires de l'Ontario; 2014 [consultation le 24 novembre 2014]. Consultable en ligne à la page : http:// www.ontario.ca/fr/lois/loi/05a11

17. Nurse-Family Partnership. Nurse-Family Partnership model elements [Internet] Denver (CO): Nurse-Family Partnership National Service Office [consultation le 24 novembre 2014]. Consultable en ligne à la page : http://www.nursefamilypartnership. org/communities/model-elements. 
18. Jack SM, Busser LD, Sheehan D, Gonzalez A, Zwygers EJ, MacMillan HL. Adaptation and implementation of the Nurse-Family Partnership in Canada. Can J Public Health. 2012;103(Suppl 1)S42-S48.

19. Ayala GX, Elder JP. Qualitative methods to ensure acceptability of behavioral and social interventions to the target population. J Public Health Dent. 2011;71(01): S69-S79.

20. Landy CK, Jack SM, Wahoush O, Sheehan D, MacMillan HL, NFP Hamilton Research Team. Mothers' experiences in the NurseFamily Partnership program: a qualitative case study. BMC Nurs. 2012;11:15.

21. British Columbia Ministry of Health and Ministry of Children and Family Development. Healthy minds, healthy people: a tenyear plan to address mental health and substance use in British Columbia. Victoria (BC): Government of British Columbia; 2010.

22. Promote, protect, prevent: our health begins here. B.C.'s guiding framework for public health [Internet]. Victoria (BC): Ministry of Health; 2013 [consultation le 24 novembre 2014]. Consultable en ligne à la page: http:// www2.gov.bc.ca/gov/content/health/aboutbc-s-health-care-system/health-priorities/bcs-guiding-framework-for-public-health

23. Haynes L, Service O, Goldacre G, Torgerson D. Test, learn, adapt: developing public policy with randomized controlled trials. London (UK): Cabinet Office Behavioural Insights Team; 2012.

24. Craig P, Dieppe P, Macintyre S, Michie S, Nazareth I, Petticrew M. Developing and evaluating complex interventions: new guidance. London (UK): Medical Research Council; 2008. PDF téléchargeable à partir du lien : http://www.mrc.ac.uk/documents/ pdf/complex-interventions-guidance/

25. Creswell JW, Plano Clark VL. Designing and conducting mixed methods research ( $2^{\mathrm{e}}$ éd.). Thousand Oaks (CA) : Sage Publications; 2011.

26. Felitti VJ, Anda RF, Nordenberg D et collabRelationship of childhood abuse and household dysfunction to many of the leading causes of death in adults. The Adverse Childhood Experiences (ACE) Study. Am J Prev Med.1998;14(4):245-258.
27. Shonkoff JP, Garner AS. The Committee on Psychosocial Aspects of Child and Family Health. The lifelong effects of early childhood adversity and toxic stress. Pediatrics. 2012;129:e232-e246.

28. Gunnar MR, Donzella B. Social regulation of the cortisol levels in early human development. Psychoneuroendocrinology. 2002; 27:199-220.

29. Repetti RL, Taylor SE, Seeman TE. Risky families: family social environments and the mental and physical health of offspring. Psychol Bull. 2002;128(2):330-66.

30. Danese A, McEwen BS. Adverse childhood experiences, allostatic, allostatic load, and age-related disease. Physiol Behav. 2012; 106(1):29-39.

31. Taylor SE, Karlamangla AS, Friedman EM, Seeman TE. Early environment affects neuroendocrine regulation in adulthood. Soc Cogn Affect Neurosci. 2011;6(2):244-251.

32. Shonkoff JP. Leveraging the biology of adversity to address the roots of disparities in health and development. Proc Natl Acad Sci U S A. 2012;109(2):17302-17307. 